\title{
AN ALTERNATE CAYLEY-DICKSON PRODUCT
}

\author{
JOHN W. BALES
}

\begin{abstract}
Although the Cayley-Dickson algebras are twisted group algebras, little attention has been paid to the nature of the CayleyDickson twist. One reason is that the twist appears to be highly chaotic and there are other interesting things about the algebras to focus attention upon. However, if one uses a doubling product for the algebras different from yet equivalent to the ones commonly used, and if one uses a numbering of the basis vectors different from the standard basis a quite beautiful and highly periodic twist emerges. This leads easily to a simple closed form equation for the product of any two basis vectors of a Cayley-Dickson algebra.
\end{abstract}

\section{INTRODUCTION}

The purpose of this paper is to give a closed form formula for the product of any two basis vectors of a Cayley-Dickson algebra.

The complex numbers are constructed by a doubling product on the set of real numbers:

$$
(a, b)(c, d)=(a c-b d, a d+b c)
$$

To produce the quaternions by a doubling product on the complex numbers requires that one take conjugation into consideration in such a way that, for real numbers the product reduces to the one above.

There are eight (and only eight) distinct Cayley-Dickson doubling products [4] which accomplish this. For each of the eight, the conjugate of an ordered pair $(a, b)$ is defined recursively by

$$
(a, b)^{*}=\left(a^{*},-b\right)
$$

The eight doubling products are:

$$
\begin{aligned}
& P_{0}:(a, b)(c, d)=\left(c a-b^{*} d, d a^{*}+b c\right) \\
& P_{1}:(a, b)(c, d)=\left(c a-d b^{*}, a^{*} d+c b\right) \\
& P_{2}:(a, b)(c, d)=\left(a c-b^{*} d, d a^{*}+b c\right) \\
& P_{3}:(a, b)(c, d)=\left(a c-d b^{*}, a^{*} d+c b\right)
\end{aligned}
$$




\section{AN ALTERNATE CAYLEY-DICKSON PRODUCT}

$$
\begin{aligned}
& P_{0}^{\top}:(a, b)(c, d)=\left(c a-b d^{*}, a d+c^{*} b\right) \\
& P_{1}^{\top}:(a, b)(c, d)=\left(c a-d^{*} b, d a+b c^{*}\right) \\
& P_{2}^{\top}:(a, b)(c, d)=\left(a c-b d^{*}, a d+c^{*} b\right) \\
& P_{3}^{\top}:(a, b)(c, d)=\left(a c-d^{*} b, d a+b c^{*}\right)
\end{aligned}
$$

Only two of these eight, $P_{3}$ and $P_{3}^{\top}$ have been investigated. The eight algebras resulting from these products are isomorphic [4] and all have the same elements and the same unit basis vectors $e_{0}, e_{1}, e_{2}, \ldots, e_{n}, \ldots$ The basis vectors will be defined below. The eight products may be arranged in four transpose pairs $P_{0}, P_{0}^{\top}, P_{1}, P_{1}^{\top}, P_{2}, P_{2}^{\top}, P_{3}, P_{3}^{\top}$. They are transposes in the sense that, given two basis vectors $e_{p}, e_{q}$, it is the case that $P\left(e_{p}, e_{q}\right)=P^{\top}\left(e_{q}, e_{p}\right)$. This holds for each of the four product pairs. So the multiplication table of the basis vectors for a product $P$ is the transpose of the multiplication table of its transpose $P^{\top}$. Given a basis vector $e_{p}$ and a basis vector $e_{q}$ there is only one $r$ for which it is the case that either $P\left(e_{p}, e_{q}\right)=e_{r}$ or $P\left(e_{p}, e_{q}\right)=-e_{r}$. For any $p$ and $q$ the value of $r$ will be the same for all eight of the products and is denoted by $p \oplus q$ (which happens to also equal $q \oplus p$ ), but whether the product of $e_{p}$ and $e_{q}$ is $e_{p \oplus q}$ or $-e_{p \oplus q}$ will depend upon which of the eight products is used.

Let $W$ denote the set of non-negative integers. For each of the eight products there is a corresponding twist function $[7,10,11] \omega: W \times W \rightarrow$ $\{-1,1\}$ such that for each $p, q \in W, P\left(e_{p}, e_{q}\right)=\omega(p, q) e_{p \oplus q}$.

Historically, researchers have been focused on the properties of the CayleyDickson algebras and not on the nature of the twist $\omega$. One reason for this is that there seemed little rhyme or reason to $\omega$. The fact that different researches numbered the basis vectors differently did not help the situation. Furthermore, for $P_{3}$ and $P_{3}^{\top}$ the function $\omega$ is particularly inscrutable. However, in [3] a heuristic Cayley-Dickson tree method was described for computing $\omega(p, q)$ for the product $P_{3}$.

In [4] the products $P_{0}, P_{0}^{\top}, P_{1}, P_{1}^{\top}, P_{2}$, and $P_{2}^{\top}$ were derived. Further investigation has shown that for the product $P_{2}$ (and its corresponding transpose) there is a simple closed form formula for $\omega$. That is the subject of this paper.

\section{BACKGROUND}

Each real number $x$ is identified with the infinite sequence $x, 0,0, \ldots$ and an ordered pair of two infinite sequences $x=x_{0}, x_{1}, x_{2}, x_{3}, \ldots$ and $y=y_{0}, y_{1}, y_{2}, y_{3}, \ldots$ is equated with the shuffled sequence

$$
(x, y)=x_{0}, y_{0}, x_{1}, y_{1}, x_{2}, y_{2}, \ldots
$$

MISSOURI J. OF MATH. SCI., SPRING 2016 


\section{JOHN W. BALES}

Only real number sequences terminating in a string of zeros are considered, that is, finite real sequences.

The basis for this space is chosen to be

$$
\begin{aligned}
& e_{0}=1,0,0,0, \ldots \\
& e_{1}=0,1,0,0,0, \ldots \\
& e_{2}=0,0,1,0,0,0, \ldots \\
& \vdots
\end{aligned}
$$

This basis differs from bases commonly used by other researchers. To distinguish this basis from others we call it the 'shuffle basis.' The shuffle basis vectors satisfy

$$
\begin{aligned}
e_{0} & =1 \\
e_{2 k} & =\left(e_{k}, 0\right) \\
e_{2 k+1} & =\left(0, e_{k}\right) .
\end{aligned}
$$

The conjugate of a sequence $x$ is $x^{*}=x_{0},-x_{1},-x_{2},-x_{3}, \ldots$ Thus,

$$
(x, y)^{*}=\left(x^{*},-y\right) .
$$

If $p, q<2^{N}$ are positive integers, let $p \oplus q$ denote the 'bit-wise exclusive or' of the binary representations of $p$ and $q$. This is equivalent to the sum of $p$ and $q$ in $\mathbb{Z}_{2}^{N}$.

The non-negative integers are an abelian group with respect to the operation $\oplus$ with identity 0 .

The twist functions for each of the eight doubling products satify the following [4].

$$
\begin{aligned}
e_{p} e_{q} & =\omega(p, q) e_{p \oplus q} \\
\omega(p, 0) & =1 \\
\omega(0, q) & =1 \\
\omega(p, p) & =-1 \text { for } p>0 \\
\omega(p, q) & =-\omega(q, p) \text { provided } 0 \neq p \neq q \neq 0 .
\end{aligned}
$$

\section{Properties of $\omega_{2}$}

The following properties of $\omega$ are peculiar to the product $P_{2}$.

$$
\begin{aligned}
& \text { If } 2^{N} \leq p<q<2^{N+1} \text { then } \omega_{2}(p, q)=1 . \\
& \text { If } 2^{N} \leq p<2^{N+1} \leq q \text { then } \omega_{2}(p, q)=(-1)^{\left\lfloor q / 2^{N}\right\rfloor} .
\end{aligned}
$$


AN ALTERNATE CAYLEY-DICKSON PRODUCT

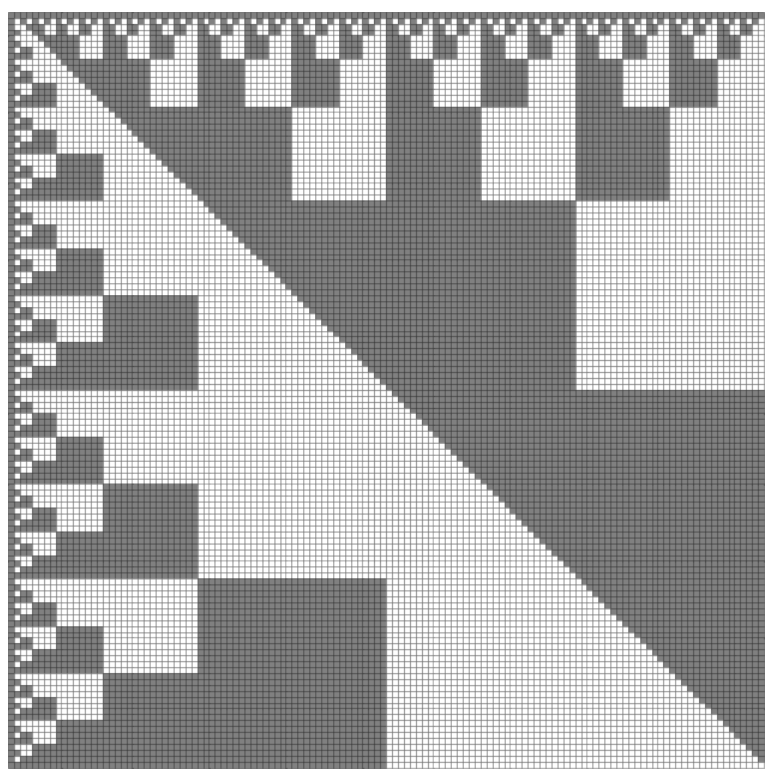

Figure 1. $\omega$ for $\mathbb{Z}_{2}^{7} \times \mathbb{Z}_{2}^{7}$.

Figure 1 shows $\omega_{2}(p, q)$ for $\mathbb{Z}_{2}^{7} \times \mathbb{Z}_{2}^{7}$. The rows and columns of the matrix are numbered 0 through 127 with gray cells representing $\omega(p, q)=1$ and white cells representing $\omega(p, q)=-1$.

\section{Traversing the Cayley-Dickson $\omega_{2}$ Tree}

In order to validate equations (3.1) and (3.2) we will traverse the $\omega$-tree [3] in Figure 2 associated with the doubling product $P_{2}$. Others have used such $\omega$ tree maps to research properties of Cayley-Dickson algebras [8].

In order to find $\omega_{2}(p, q)$ for non-negative integers $p$ and $q$ it will be necessary to shuffle their bits. In order not to confuse this process with the shuffling $(x, y)$ of sequences $x$ and $y$, the shuffle of integers $p$ and $q$ will be denoted using square brackets. So, for example, $[111,101]=11,10,11$ and $[11,11101]=[00011,11101]=01,01,01,10,11$. Notice that the shuffled binary numbers have been rendered as a sequence of binary doublets. Each doublet, beginning with the leftmost, is an instruction for traversing the $\omega_{2}$-tree beginning with the top $\mathbf{C}$ node. A 0 is an instruction to move down a left branch and a 1 is an instruction to move down a right branch of the tree. 


\section{JOHN W. BALES}

To find $\omega_{2}(p, q)$ by this method, traverse the tree using instruction sequence $[p, q]$. Terminating at -1 or $-\mathbf{D}$ means that $\omega(p, q)=-1$. Terminating at any other node means that $\omega_{2}(p, q)=1$. An important property of $\omega_{2}$ is that once either 1 or -1 is reached it is unnecessary to continue traversing the tree and it will always be the case that $\omega_{2}(p, q)=1$ or $\omega_{2}(p, q)=-1$, respectively.

As an example of how one traverses the $\omega_{2}$-tree, let us find the basis vector product $e_{3} e_{14}$. First, $3=0011_{B}$ and $14=1110_{B}$. So $3 \oplus 14=$ $1101_{B}=13$. So $e_{3} e_{14}=\omega_{2}(3,14) e_{13}$. Now $[3,14]=01,01,11,10$. Using this sequence of doublets to traverse the $\omega$-tree gives us $\mathbf{T}, \mathbf{T},-1,-1$. So $e_{3} e_{14}=-e_{13}$. One may stop, of course, with the first -1 encountered.

Next, it will be seen how to use the $\omega_{2}$-tree in Figure 2 to validate equations (3.1) and (3.2).

Begin with equation (3.1), suppose $2^{N} \leq p<q<2^{N+1}$. Then $[p, q]=$ $11, \ldots$. The doublets following the first will be either $00,01,10$, or 11 . The first doublet 11 moves to node - D. Subsequent doublets of either 00 or 11 remain at node $-\mathbf{D}$. Since $p<q$ there must occur a doublet 01 and it must occur prior to any potential doublet 10 . But 01 moves from node $-\mathbf{D}$ to node 1 . Thus, $\omega_{2}(p, q)=1$ verifying equation (3.1).

For equation (3.2) suppose $2^{N} \leq p<2^{N+1} \leq q$. Then it is either the case that $[p, q]=01, \ldots, 10, \ldots$ or it is the case that $[p, q]=01, \ldots, 11, \ldots$ (where the 10 and 11 doublets are the bits of $p$ and $q$ corresponding to $2^{N}$ ). In either case the first ellipsis consists of binary doublets of the form 00 or 01 so we are at a $\mathbf{T}$ node until arriving at either the doublet 10 in which case $\omega(p, q)=1$ or we arrive at the doublet 11 in which case $\omega(p, q)=-1$. In the first case, $\left\lfloor q / 2^{N}\right\rfloor$ is even and in the second case $\left\lfloor q / 2^{N}\right\rfloor$ is odd. So in either case $\omega(p, q)=(-1)^{\left\lfloor q / 2^{N}\right\rfloor}$ verifying equation (3.2).

Let us illustrate the use of equations (3.1) and (3.2) with a couple of examples.

Find $e_{35} e_{55}$.

Since $35=100011_{B}$ and $55=110111_{B}$ then $35 \oplus 55=10100_{B}=20$. So $e_{35} e_{55}=\omega_{2}(35,55) e_{20}$. And since $2^{5} \leq 35<55<2^{6}$ it follows from equation 3.2 that $\omega_{2}(35,55)=1$. So $e_{35} e_{55}=e_{20}$.

Find $e_{87} e_{340}$.

Convert $87=001010111_{B}$ and $340=101010100_{B}$, so $87 \oplus 340=$ $100000011_{B}=259$. So $e_{87} e_{340}=\omega_{2}(87,340) e_{259}$. Since $64 \leq 87<128$, and $128 \leq 340$ and $\left\lfloor\frac{340}{64}\right\rfloor=5$ then $\omega_{2}(87,340)=(-1)^{5}=-1$. So $e_{87} e_{340}=-e_{259}$

Find $e_{51} e_{12}$.

First, $e_{51} e_{12}=-e_{12} e_{51} .12=001100_{B}$ and $51=110011_{B}$ so $12 \oplus 51=$ $111111_{B}=63$. So $e_{51} e_{12}=-e_{12} e_{51}=-\omega_{2}(12,51) e_{63}$. Since $8 \leq 12<$ $16 \leq 51$ and $\left\lfloor\frac{51}{8}\right\rfloor=6, \omega_{2}(12,51)=(-1)^{6}=1$. So $e_{51} e_{12}=-e_{63}$. 


\section{AN ALTERNATE CAYLEY-DICKSON PRODUCT}

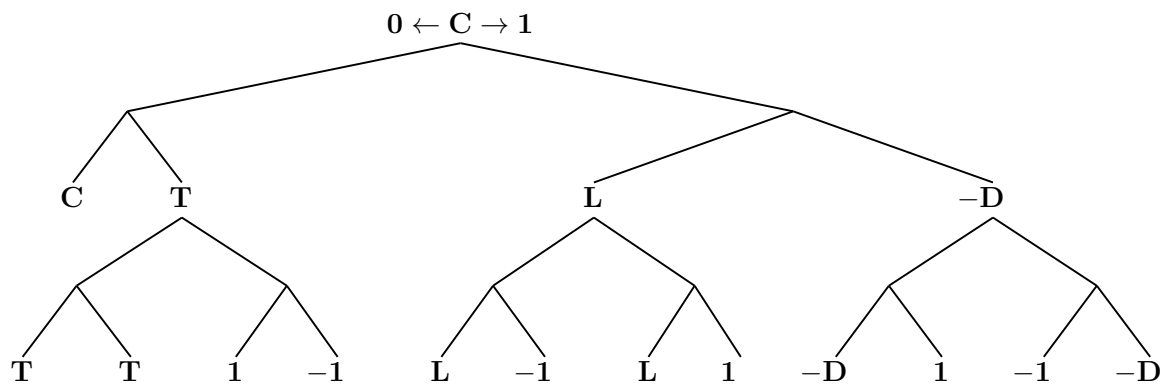

FiguRE 2. Twist tree for $\omega_{2}$.

Lest the reader get eye strain from trying to verify the results for $\mathbb{Z}_{2}^{7} \times \mathbb{Z}_{2}^{7}$ using Figure 1 on page 91, the $\omega_{2}$ table for $\mathbb{Z}_{2}^{5} \times \mathbb{Z}_{2}^{5}$ is provided in Figure 3 on page 94 . Recall that gray cells denote $\omega_{2}(p, q)=1$ and white cells denote $\omega_{2}(p, q)=-1$.

To give a better indication of the fractal nature of $\omega_{2}$, Figure 4 is the $1024 \times 1024$ bit-mapped image of $\omega_{2}$ for $\mathbb{Z}_{2}^{10} \times \mathbb{Z}_{2}^{10}$. For comparison we also provide the corresponding image of the 'inscrutable' $\omega_{3}$ for $\mathbb{Z}_{2}^{10} \times \mathbb{Z}_{2}^{10}$ in Figure 5 to give a visual indication of why no one has searched for a simple formula for it.

\section{Conclusion}

The problem historically with finding a simple closed form equation for the product of two Cayley-Dickson basis vectors has been caused by various approaches to the algebras. One issue is that only two of the eight CayleyDickson doubling products have been used $[12,6,2,3,5]$ each of which is the transpose of the other.

$$
\begin{aligned}
& (a, b)(c, d)=\left(a c-d b^{*}, a^{*} d+c b\right) . \\
& (a, b)(c, d)=\left(a c-d^{*} b, d a+b c^{*}\right) .
\end{aligned}
$$

Unfortunately, the $\omega$ matrix of these two is sufficiently chaotic to dissuade further investigation. Furthermore, a different way of numbering the basis vectors has traditionally been used which further scrambles the $\omega$ matrix. These issues have conspired to inhibit investigation into $\omega$.

Now we see that if the doubling product

$$
P_{2}:(a, b)(c, d)=\left(a c-b^{*} d, d a^{*}+b c\right)
$$

is used and if the basis vectors are indexed over the group $(W, \oplus)$ (the 'shuffle' basis) a natural inverse fractal pattern emerges leading to the simple result in the following theorem. 
JOHN W. BALES

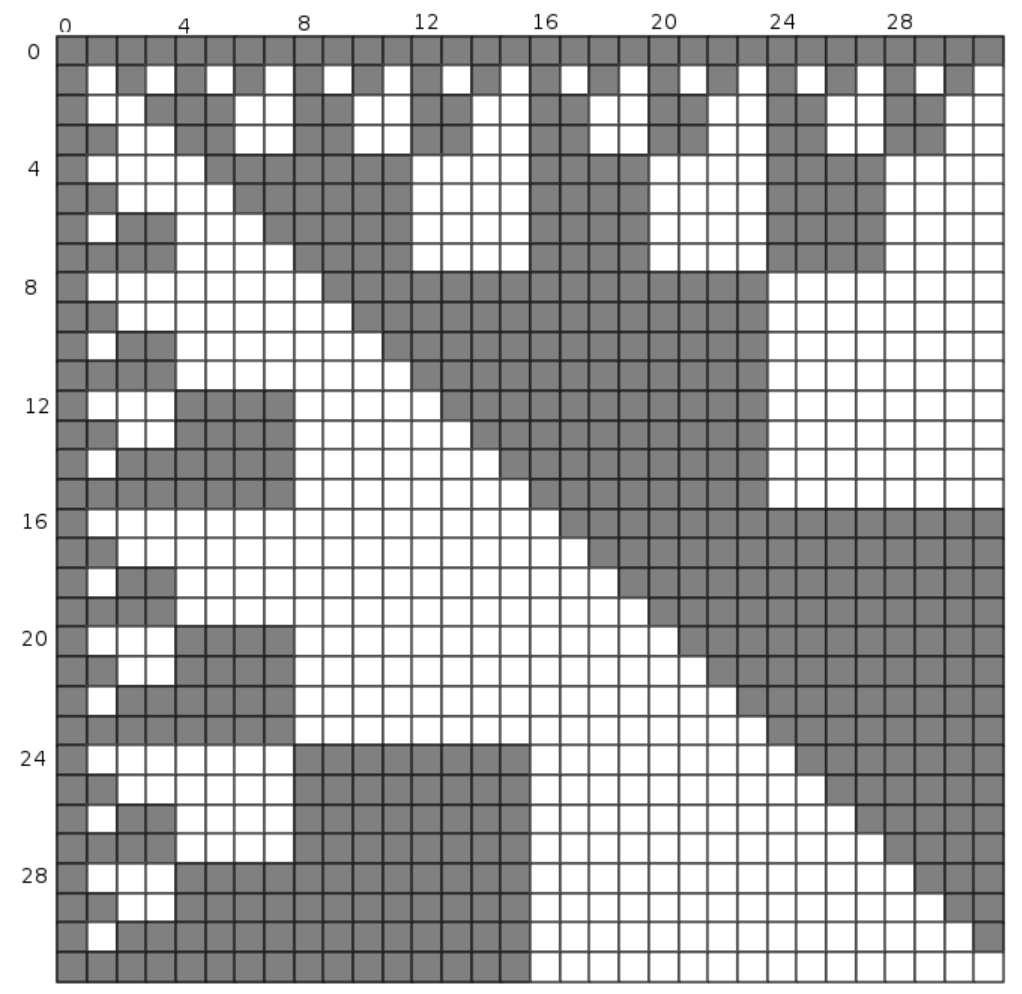

Figure 3. $\omega_{2}$ for $\mathbb{Z}_{2}^{5} \times \mathbb{Z}_{2}^{5}$.

Theorem 5.1. If $2^{N} \leq p<q<2^{N+1}$ then $e_{p} e_{q}=e_{p \oplus q}$.

If $2^{N} \leq p<2^{N+1} \leq q$ then $e_{p} e_{q}=(-1)^{\left\lfloor q / 2^{N}\right\rfloor} e_{p \oplus q}$.

Combined with $e_{0}=1, e_{p}^{2}=-1$ for $p>0$ and $e_{p} e_{q}=-e_{q} e_{p}$ for $0 \neq p \neq q \neq 0$ we have a simple closed formulation for the product of any two Cayley-Dickson basis vectors. 
AN ALTERNATE CAYLEY-DICKSON PRODUCT

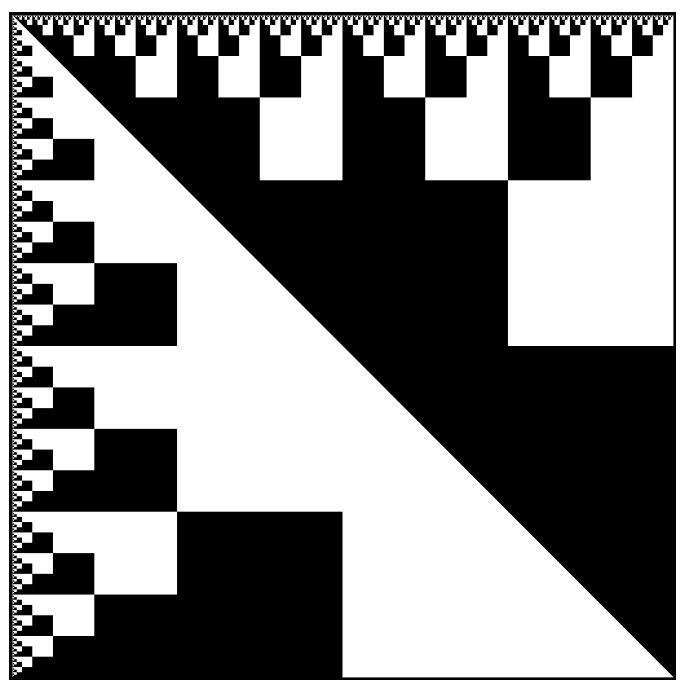

FiguRE 4. $\omega_{2}$ for $\mathbb{Z}_{2}^{10} \times \mathbb{Z}_{2}^{10}$.

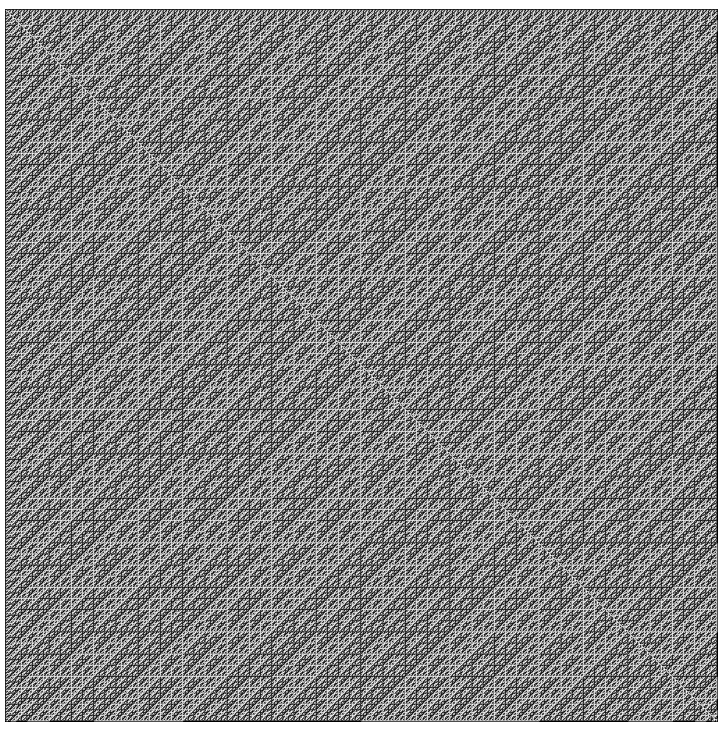

FiguRE 5 . $\omega_{3}$ for $\mathbb{Z}_{2}^{10} \times \mathbb{Z}_{2}^{10}$.

REFERENCES

[1] W. Ambrose, Structure theorems for a special class of Banach algebras, Trans. Amer. Math. Soc., 57 (1945), 364-386.

MISSOURI J. OF MATH. SCI., SPRING 2016 


\section{JOHN W. BALES}

[2] J. Baez, The Octonions, Bul. Am. Math. Soc., 39.2 (2001), 145-205.

[3] J. Bales, A tree for computing the Cayley-Dickson twist, Missouri J. of Math. Sci., 21.2 (2009), 83-93.

[4] J. Bales, The eight Cayley-Dickson doubling products, Adv. in Appl. Clifford Alg., 25.4 (2015), 1-23.

[5] D. Biss, J. Christensen, D. Dugger, and D. Isaksen, Eigentheory of Cayley-Dickson algebras in Forum Mathematicum, 21.5 (2009), 833-851.

[6] R. Brown, On generalized Cayley-Dickson algebras, Pacific J. Math., 20.3 (1967), $415-422$.

[7] R. Busby and H. Smith, Representations of twisted group algebras, Trans. Am. Math. Soc., 149.2 (1970), 503-537.

[8] C. Flaut and V. Shpakivskyi, Holomorphic Functions in Generalized CayleyDickson Algebras, Springer Basel, Adv. in Appl. Clifford Algebras, 25.1 (2015), 95-112.

[9] L. E. Dickson, On quaternions and their generalization and the history of the eight square theorem, Annals of Mathematics 20.3 (1919), 155-171.

[10] C. M. Edwards and J. T. Lewis, Twisted group algebras I, Communications in Mathematical Physics, 13.2 (1969), 119-130.

[11] W. F. Reynolds, Twisted group algebras over arbitrary fields, Illinois J. Math., 15.1 (1971), 91-103.

[12] R. Schafer, On the algebras formed by the Cayley-Dickson process, Amer. J. Math., 76 (1954), 435-446.

MSC2010: 16S99, 16W99

Key words and phrases: Cayley-Dickson, doubling product, twisted group product, fractal, twist tree

Department of Mathematics, Tuskegee University, Tuskegee, AL 36088

E-mail address: john.w.bales@gmail.com 